\title{
Communautés végétales et diversité des chiroptères dans les forêts de Niaouli et de Lokoli au Sud du Bénin
}

\author{
B. A. DJOSSA ${ }^{1,3^{*}}$, A. C. ADOMOU ${ }^{2}$ et B. A. $\operatorname{SINSIN}^{1}$ \\ ${ }^{1}$ Laboratoire d'Ecologie Appliquée - FSA/UAC, Bénin. \\ ${ }^{2}$ Département de biologie végétale - FAST/UAC, Bénin. \\ ${ }^{3}$ Ecole Nationale Supérieure des Sciences et Techniques Agronomiques de Kétou- ENSTA/UAC, Bénin. \\ *Auteur correspondant, E-mail : djossabruno@gmail.com
}

\section{RESUME}

La présente étude a évalué l'influence du type d'habitats sur la diversité et la structure des communautés des chiroptères dans les forêts de Niaouli et de Lokoli situées au Sud du Bénin. L'inventaire floristique est réalisé avec la méthode de Braun-Blanquet et celui des chiroptères est fait au filet japonais. Ces deux formations forestières ont montré une dissemblance sur le plan floristique avec une dissimilarité de 0,89 et au niveau des communautés de chiroptères qui y sont inféodées avec une dissimilarité de 0,61 . Une DCA a aussi montré que les deux formations forestières constituent deux écosystèmes différents de même que les communautés des chiroptères associées. La majorité des espèces de chiroptères recensées dans ces deux forêts sont des espèces dépendantes des forêts denses humides. Mimetellus moloneyi (Thomas 1891), Hipposideros fuliginosus (Temminck 1853) et Hipposideros tephrus (Cabrera 1906) rapportées sont nouvelles pour le Bénin. Les espèces communes aux deux écosystèmes sont caractéristiques des milieux dégradés. La présence des trois nouvelles espèces de chiroptère dans ces forêts de Niaouli et de Lokoli montre qu'elles constituent deux îlots de forêts denses humides dans cette région du pays et doivent être conservés pour les générations actuelles et futures.

(C) 2010 International Formulae Group. All rights reserved.

Mots clés: Forêt, chiroptère, diversité, structure de communauté, conservation, Bénin.

\section{INTRODUCTION}

Les pressions constantes sur les ressources naturelles dues à l'utilisation des terres constituent une des causes majeures de la fragmentation et des pertes continues d'habitats (Henle, 2004). La FAO a rapporté récemment pour le Bénin une perte annuelle de 65000 ha. Ces dégradations imputables à l'homme sont souvent de nature agropastorales (Houinato et Sinsin, 2000). Les pertes et fragmentations d'habitats sont probablement les causes majeures d'extinction d'espèces dans ces dernières décennies (Henle et Streit, 1990). Une perturbation des écosystèmes naturels pourrait affecter la qualité de l'habitat, mais aussi la disponibilité spatio-temporelle des ressources alimentaires pour les animaux qui vivent dans ces écosystèmes. En ce qui concerne les chiroptères en particulier, Fenton (1997) et Pierson (1998) ont identifié quelques éléments/conditions pour les conserver, à savoir: la protection des habitats, la disponibilité des ressources alimentaires et de la protection des perchoirs. Plusieurs auteurs ont également rapporté que les pressions qui 
s'exercent sur les populations de chiroptères à travers le monde se résument à la perte ou fragmentation d'habitats, à la perturbation des perchoirs, aux expositions à des toxines, aux pressions de chasse et à l'introduction de prédateurs (Fenton, 1997; Pierson, 1998; Agosta, 2002). La région méridionale du Bénin qui abrite plus de la moitié de sa population totale enregistre de fortes pressions sur les ressources naturelles en générale et en particulier sur le couvert végétal avec comme corollaire des pertes sévères d'habitats naturels pour la faune sauvage. Cette situation réserve probablement des conditions peu favorables aux animaux sauvages comme les chiroptères qui ont besoins de conserver des interactions avec les plantes.

Cette étude a pour objectif d'inventorier la diversité des chiroptères dans les forêts de Niaouli et de Lokoli d'une part, et l'effet de la composition floristique desdites forêts sur la diversité des chiroptères dans cette région d'autre part.

\section{MATERIEL ET METHODES Milieu d'étude \\ Forêt marécageuse de Lokoli}

La forêt marécageuse de Lokoli est située dans l'arrondissement de Koussoukpa, Commune de Zogbodomey, Département du Zou au Bénin. La forêt se situe entre $7^{\circ} 03^{\prime} \mathrm{N}$ et $2^{\circ} 15^{\prime}$ E. Elle couvre une superficie d'environ 3000 ha et est localisée dans la partie Nord de la basse vallée de l'Ouémé en zone guinéenne (Adomou et al., 2009). La pluviométrie moyenne annuelle est de 1112 mm. L'humidité relative moyenne est de $75 \%$. La température varie entre 26 et $28{ }^{\circ} \mathrm{C}$. Le sol est argilo-limoneux, hydromorphe et tourbeux. La forêt est entourée de trois villages (Lokoli, Koussoukpa et Dèmè) avec une population totale estimée à 4810 habitants (INSAE, 2002). Les principales activités sont l'agriculture, l'artisanat, le ramassage d'escargot, l'exploitation du vin et des feuilles de Raphia hookeri.

\section{Forêt dense semi-décidue de Niaouli}

La forêt dense semi-décidue de Niaouli se situe dans le village qui lui a donné son nom, et qui abrite depuis 1904 la Station de recherche sur les cultures vivrières dont dépend ladite forêt. La forêt de Niaouli est située dans l'arrondissement d'Atogon, Commune d'Allada et couvre une superficie d'environ 220 ha. De nos jours, environ 65,5 ha représentent les derniers vestiges de forêt dense semi-décidue qui couvrait le Sud Bénin (Djossa et al., 2008). Elle se situe géographiquement entre $6^{\circ} 44^{\prime} \mathrm{N}$ et $2^{\circ} 08^{\prime} \mathrm{E}$. Une partie de cette forêt dénommée « forêt du bas fond » couvre 24,2 ha et qui est périodiquement inondée par le ruisseau Ava. Une seconde portion dénommée «forêt du plateau » couvre 41,3 ha et est représentée par une forêt dense humide semi-décidue de terre ferme. La pluviométrie moyenne annuelle est de $1165 \mathrm{~mm}$. L'humidité relative moyenne est de $73 \%$. La température moyenne mensuelle varie entre 26,8 et $28{ }^{\circ} \mathrm{C}$ avec une amplitude thermique de $9,6{ }^{\circ} \mathrm{C}$. Le sol est ferralilitique, profond et bien drainé dans l'ensemble avec une portion de sol hydromorphe dans le basfond. La population riveraine est estimée à 2000 habitants (INSAE, 2002) composée surtout des Aizo et qui pratiquent surtout l'agriculture.

\section{Méthodes \\ Données floristiques}

Les points d'inventaire floristique sont choisis dans la forêt dense humide qui a un recouvrement arborescent d'au moins 40\% (Figure 2). Les relevés phytosociologiques sont effectués dans des placeaux de 30 × $30 \mathrm{~m}$ selon la méthode classique de Braun-Blanquet (1932) ; 11 et 08 relevés floristiques ont été respectivement réalisés à Lokoli et à Niaouli. Le diamètre à hauteur d'homme ( $\mathrm{dbh} \geq 10$ $\mathrm{cm}$ ) à 1,30 m du sol ou à $30 \mathrm{~cm}$ au-dessus des contreforts des ligneux est mesuré à l'aide d'un ruban pi. Les coordonnées géographiques des placettes sont prises à l'aide d'un GPS (Global Positioning System). Les espèces sont identifiées à l'aide de la Flore Analytique du Bénin (Akoègninou et al., 2006) et le Guide de terrain des ligneux des forêts denses humides de l'Afrique de l'Ouest (Hawthorne et Jongkind, 2006). 


\section{Inventaire des chiroptères}

L'inventaire des chiroptères a été fait avec des filets japonais du 18 au 21 décembre 2008 à Lokoli avec 5 filets par nuit (240 heures d'ouverture de filet) et du 07 au 09 avril 2009 à Niaouli avec 5 filets par nuit (180 heures d'ouverture de filet). Le filet japonais (R. Volwinkel, Velbert, Germany) mesurant $12 \mathrm{~m} \mathrm{x} 2,8 \mathrm{~m}$ avec 5 étages ( $16 \mathrm{~mm}$ mèche, 70 denier/ 2 plis). Les filets étaient ouverts entre 19:00 et 19:30 heures jusqu'á 00:00 heures et encore de 4:00 à 6:00 heures. L'identification des espèces s'est basée sur les clés dichotomiques de Hayman et Hill (1971) et de la compilation de Bergmans (2002). Les spécimens capturés étaient pesés avec des pesons $(100 \mathrm{~g}, 300 \mathrm{~g}$ et $600 \mathrm{~g}$ précision $1 \mathrm{~g})$, les avant-bras étaient mesurés avec un pied à coulisse (précision $1 \mathrm{~mm}$ ).

\section{Traitement des données}

La classification hiérarchique ascendante utilisant "cluster analysis" (méthode de Ward) a permis de faire la typologie des groupements végétaux. La diversité floristique a été appréciée en utilisant un certain nombre d'indices et de variables présentés ci-dessous.

L'indice de Shannon (Shannon, 1948) qui intègre à la fois la richesse spécifique et l'abondance relative des espèces. Il est utile car sa valeur augmente non seulement en fonction du nombre d'espèces, mais aussi selon l'abondance relative de chaque espèce dans la communauté. L'indice est maximal quand tous les individus sont répartis d'une façon égale sur toutes les espèces (Frontier, 1983). Il est donné par la formule suivante :

$$
\mathrm{H}^{\prime}=-\sum_{i=1}^{s} p_{i} \log _{2} p_{i}
$$

avec $p_{i}=$ l'abondance relative de l'espèce $i$.

L'indice d'équitabilité de Pielou ou de régularité exprime, dans un échantillon donné, le rapport de la diversité atteinte $\left(\mathrm{H}^{\prime}\right)$ et la diversité maximale $\left(\log _{2} \mathrm{~S}\right)$ pouvant être obtenue avec le même nombre de taxons (Frontier et Pichod-Viale, 1991). Cet indice varie de 0 à 1 ; il est maximal quand les espèces ont des abondances identiques dans le peuplement et il est minimal quand une seule espèce domine tout le peuplement. Il est donné par la formule suivante :

$$
\mathrm{J}=\mathrm{H}^{\prime} / \log _{2} \mathrm{~S}
$$

avec $\mathrm{S}=$ la richesse spécifique.

Pour rendre compte de la structure démographique du peuplement ligneux, la répartition des individus par classe de diamètre est effectuée.

L'indice de Jaccard a permis de comparer la composition floristique des deux forêts. Il est défini comme suit :

$$
P_{j}=100 \times \frac{c}{a+b-c}
$$

Avec $\mathrm{P}_{\mathrm{j}}=$ Indice de similarité de Jaccard, $\mathrm{a}=$ nombre d'espèces présentes dans la communauté $\mathrm{A} ; \mathrm{b}=$ nombre d'espèces présentes dans la communauté $\mathrm{B}$, et $\mathrm{c}=$ nombre d'espèces communes aux deux communautés. Les communautés sont dites semblables lorsque cette similarité est $>0,50$.

Les abondances relatives (nombre capturé par heure d'ouverture de filet) de chacune des espèces de chiroptère calculées par écosystème ont été utilisées pour faire une DCA (Ordination plot with reciprocal averaging) dans CAP 2.15 (Community Analysis Packages version 2.15) pour apprécier le regroupement des espèces au sein des communautés des chiroptères dans un plan factoriel tant dans la forêt de Niaouli que dans celle de Lokoli. La similarité entre les deux communautés de chiroptère a aussi été testée au moyen de l'indice de Jaccard (voir formule ci-dessous). La diversité des chiroptères a été comparée et présentée avec des histogrammes construits dans Excel version 2003.

\section{RESULTATS}

Typologie des communautés végétales $\mathrm{La}$ classification hiérarchique ascendante utilisant "cluster analysis" (méthode de Ward) a permis de discriminer deux grands groupes de relevés en fonction de la topographie (Figure 2):

- Groupe de relevés effectués dans la forêt marécageuse de Lokoli (FM) qui est dominée 
par les espèces telles que Alstonia congensis, Xylopia rubescens, Syzygium owariense, Hallea ledermannii, Spondianthus preussii et Raphia hookeri.

- Groupe de relevés effectués dans la forêt dense humide semi-décidue de Niaouli (FDHSD) qui est dominée par les espèces telles que Celtis zenkeri, Triplochiton scleroxylon, Trichilia prieureana, Antiaris toxicaria et Pentaclethra macrophylla.

La valeur de l'indice de Jaccard $(0,11)$ montre que la similarité floristique entre les deux forêts est très faible.

Diversité végétale et structure de la forêt marécageuse de Lokoli

Un total de 125 espèces, appartenant à 106 genres et 55 familles sont recensées. Les familles les plus représentées en espèces sont : Rubiaceae (17 espèces, soit 14\%), Leguminosae (10 espèces, soit $8 \%)$ et Moraceae ( 9 espèces, soit $9 \%$ ). La richesse spécifique des ligneux de $\mathrm{dbh} \geq 10 \mathrm{~cm}$ est de 35 espèces. L'indice de Shannon $\left(\mathrm{H}^{\prime}=3,24\right.$ bits) est moyen alors que l'équitabilité de Pielou $(\mathrm{Eq}=0,63)$ est faible.

Le profil structural de la forêt montre 4 strates. La strate arborescente supérieure, de 15 à $30 \mathrm{~m}$, est dominée par Alstonia congensis, Xylopia rubescens, Syzygium owariense et Hallea ledermannii. La strate arborescente inférieure, de 8-15 m, comprend Spondianthus preussii, Anthocleista vogelii et Nauclea diderrichii. La strate arbustive, de 3$8 \mathrm{~m}$, est dominée par Pterocarpus santalinoides, Ficus vogeliana et Psychotria articulata. Enfin, la strate herbacée, de 0-3 m de hauteur, comporte les espèces telles que Lasiomorpha senegalensis, Cyclosorus striatus, Nephrolepis bisserata, Tacateza pedicellata, Grewia barombiensis et Lycopodium cernuum.

La densité des ligneux de $\mathrm{dbh} \geq 10 \mathrm{~cm}$ est de 620 tiges/ha. L'aire basale évaluée pour l'ensemble de la forêt est de $44,9 \mathrm{~m}^{2} /$ ha ; les espèces telles que Alstonia congensis $(35 \%)$ et Xylopia rubescens (24\%) y contribuent fortement.
La structure diamétrique du peuplement ligneux s'ajuste à une fonction logarithmique. Elle montre une tendance vers la distribution en "L" avec un bon recrutement des classes de faible diamètre vers celles plus grandes. Cette structure est caractéristique des forêts tropicales moins dégradées (Figure 3).

Diversité végétale et structure de la forêt dense humide semi-décidue de Niaouli

Un total de 168 espèces de plantes, appartenant à 145 genres et 54 familles, sont recensées. Les familles les plus représentées en espèces sont : Leguminosae (21 espèces, soit 39\%) Rubiaceae (17 espèces, soit 31\%), Apocynaceae (10 espèces, soit 18,50\%), Euphorbiaceae (10 espèces, soit 18,50\%), Sterculiaceae (7 espèces, soit 13\%), Sapindaceae (7 espèces, soit 13\%). La richesse spécifique des ligneux de $\mathrm{dbh} \geq 10$ $\mathrm{cm}$ est de 55 espèces. L'indice de Shannon $\left(\mathrm{H}^{\prime}=3,10\right.$ bits $)$ est moyen alors que l'équitabilité de Pielou $(\mathrm{Eq}=0,80)$ est faible.

Le profil structural de la forêt montre 4 strates. La strate arborescente supérieure, de 15 à $30 \mathrm{~m}$, est dominée par Celtis zenkeri, Triplochiton scleroxylon Trilepiseum madagascariense, Dialium guineense, Antiaris toxicaria, Cola gigantea, Pentaclethra macrophylla et Ceiba pentandra.

La strate arborescente inférieure, de 8$15 \mathrm{~m}$, comprend les espèces telles que, Dialium guinense, Cola millenii, Lannea nigritana et Pouteria alnifolia. La strate arbustive, de 3-8 m, est dominée par Rothmannia longiflora, Dictyandra aborescens, Monodora tenuifolia et Dichapetalum guineensis. Enfin, la strate herbacée, de 0-3 m de hauteur, comporte les espèces telles que Clausena anisata, Oplismenus hirtellus, Cyclosorus striatus, Keetia hispida et Olyra latifolia.

La densité des ligneux de $\mathrm{dbh} \geq 10 \mathrm{~cm}$ est de 607 tiges/ha. L'aire basale évaluée pour l'ensemble de la forêt est de $42 \mathrm{~m}^{2} / \mathrm{ha}$; les espèces telles que Antiaris africana (17\%) et Cola spp (11\%), Dialium spp (10\%), Celtis spp (8\%) et Triplochiton scleroxylon (6\%) sont bien représentées. 
La structure diamétrique du peuplement ligneux s'ajuste à une fonction exponentielle. Elle montre une tendance vers la distribution en "L" avec une forte mortalité (> 50\%) lorsqu'on passe de la classe de diamètre 10-20 $\mathrm{cm}$ à la classe 20-40 cm (Figure 4).

Aussi, l'indice de Jaccard a montré qu'il existe une dissemblance $(0,89)$ entre les deux forêts.

\section{Composition floristique et communautés de chiroptères}

Une prospection de 280 heures dans la forêt dense semi-décidue de Niaouli $(120 \mathrm{~h})$ et la forêt marécageuse de Lokoli (160 h) ont permis de capturer 103 spécimens de chiroptères distribués dans 4 familles, 14 genres et 18 espèces. La distribution des espèces de chiroptères par écosystème est présentée dans le Tableau 1 ci-dessous.

Ces deux écosystèmes qui couvrent seulement 3220 ha (Lokoli : 3000 ha et Niaouli : 220 ha) abritent, sur la base des présentes prospections, environ $34 \%$ de la diversité des chiroptères du Bénin connue à ce jour. Mieux, trois espèces (Mimetellus moloneyi, Hipposideros fuliginosus et Hipposideros tephrus) recensées sont nouvelles pour la liste des espèces de chiroptères connus pour le Bénin à ce jour. La forêt de Lokoli a contribué pour $75 \%$ à cette augmentation de diversité.

La diversité des familles de chiroptères est aussi présentée sur la Figure 5 qui suit : $50 \%$ des familles connues au Bénin à ce jour ont été recensées avec les prospections actuelles de ces deux écosystèmes. Les familles des Vespertilionidae et des Molossidae étaient plus diversifiées dans la forêt de Lokoli que dans la forêt de Niaouli.

La diversité spécifique des chiroptères comparée entre les forêts de Lokoli et de Niaouli est présentée sur la Figure 6 qui suit :

Environ $39 \%$ de l'ensemble des espèces recensées au niveau des deux forêts a été rapporté seulement de la forêt de Lokoli, contre $22 \%$ de la forêt de Niaouli.

L'indice de similarité de Jaccard a permis de révéler que les deux forêts étudiées étaient différentes de part la diversité des chiroptères qui $\mathrm{y}$ sont inféodés. La dissemblance était de 0,61 entre Niaouli et Lokoli.

Typologie des communautés de chiroptères en relation avec les habitats

La structure et la composition des communautés de chiroptères semblent différentes entre la forêt dense semi-décidue de Niaouli et de la forêt marécageuse de Lokoli. Les résultats sont présentés sur la Figure 7 ci-dessous.

L'axe 1 semble porter un gradient de diversité plaçant la forêt de Niaouli moins diversifiée dans le plan négatif et la forêt de Lokoli plus diversifiée dans le plan positif allant vers la pointe dudit axe. L'axe 2 par contre semble porter un gradient d'humidité en positionnant la communauté dominée par les espèces capturées uniquement à Niaouli à l'extrémité négative de l'axe et celle dominée par les espèces capturées seulement dans Lokoli à l'extrémité positive dudit axe. Les espèces communes aux deux écosystèmes étant positionnées au milieu des deux communautés extrêmes et autour de l'origine des axes.

\section{DISCUSSION}

\section{Type d'habitats et communautés des chiroptères}

La diversité et les communautés de chiroptères rapportées au cours de la présente étude de la forêt de Niaouli sont différentes de celles obtenues dans la forêt marécageuse de Lokoli. Hodgkison et al. (2004) avaient déjà rapporté de la Malaisie, l'influence de la structure de l'habitat sur la composition des communautés des chiroptères. En effet, la composition floristique n'est pas identique entre les deux forêts étudiées comme le montre la typologie obtenue avec le dendrogramme qui a séparé nettement lesdites forêts. Aussi, si dans la forêt marécageuse de Lokoli les deux premières classes de diamètre $(10-20 \mathrm{~cm}$ et $20-30 \mathrm{~cm})$ sont bien fournies, la forêt de Niaouli n'en présente pas autant alors que cette dernière présente plus de sujets de grand diamètre. 


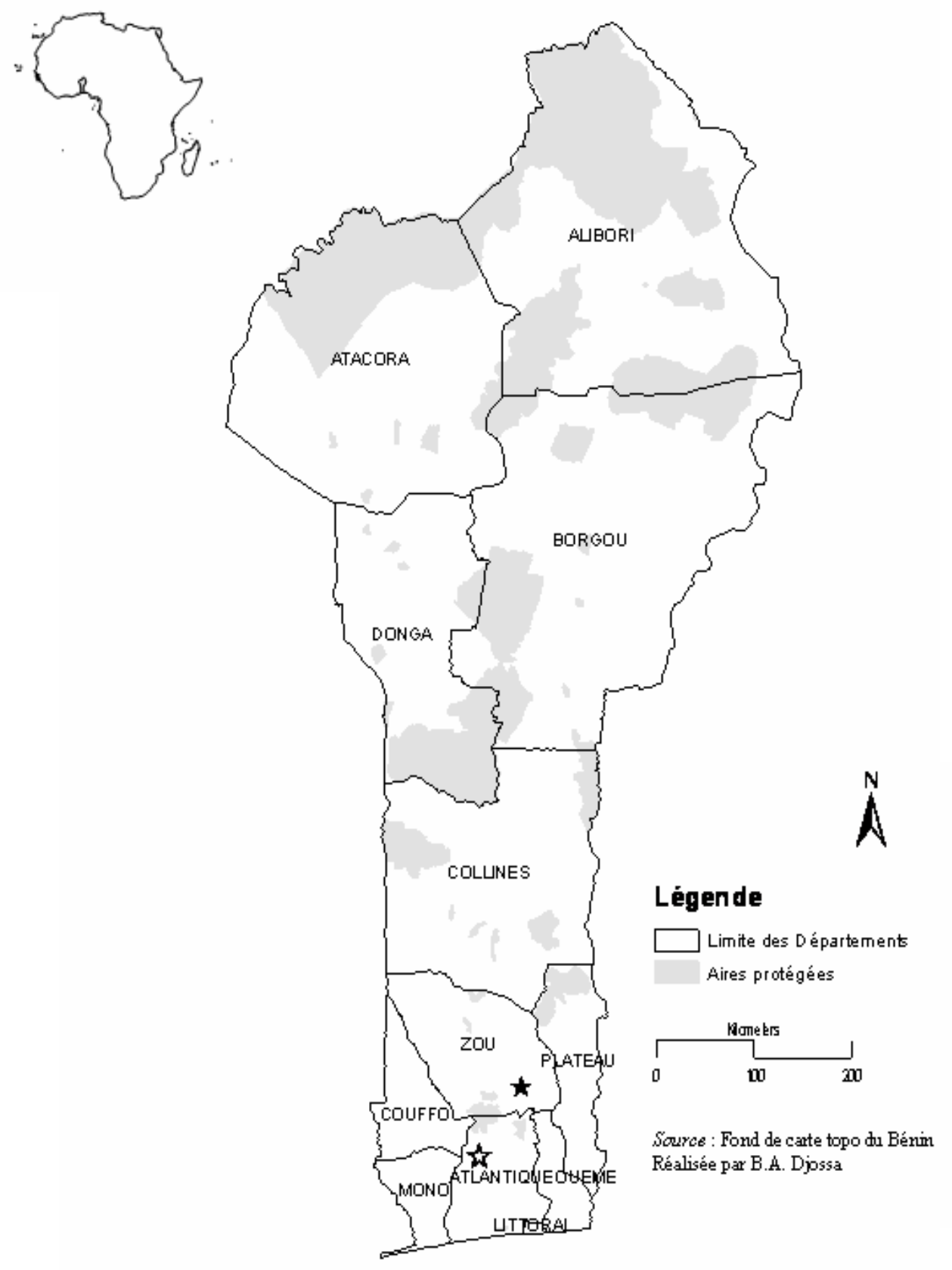

Figure 1 : Carte du Bénin montrant la localisation de la forêt marécageuse de Lokoli (étoile noire pleine) et de la forêt dense semi-décidue de Niaouli (étoile noire vide). 


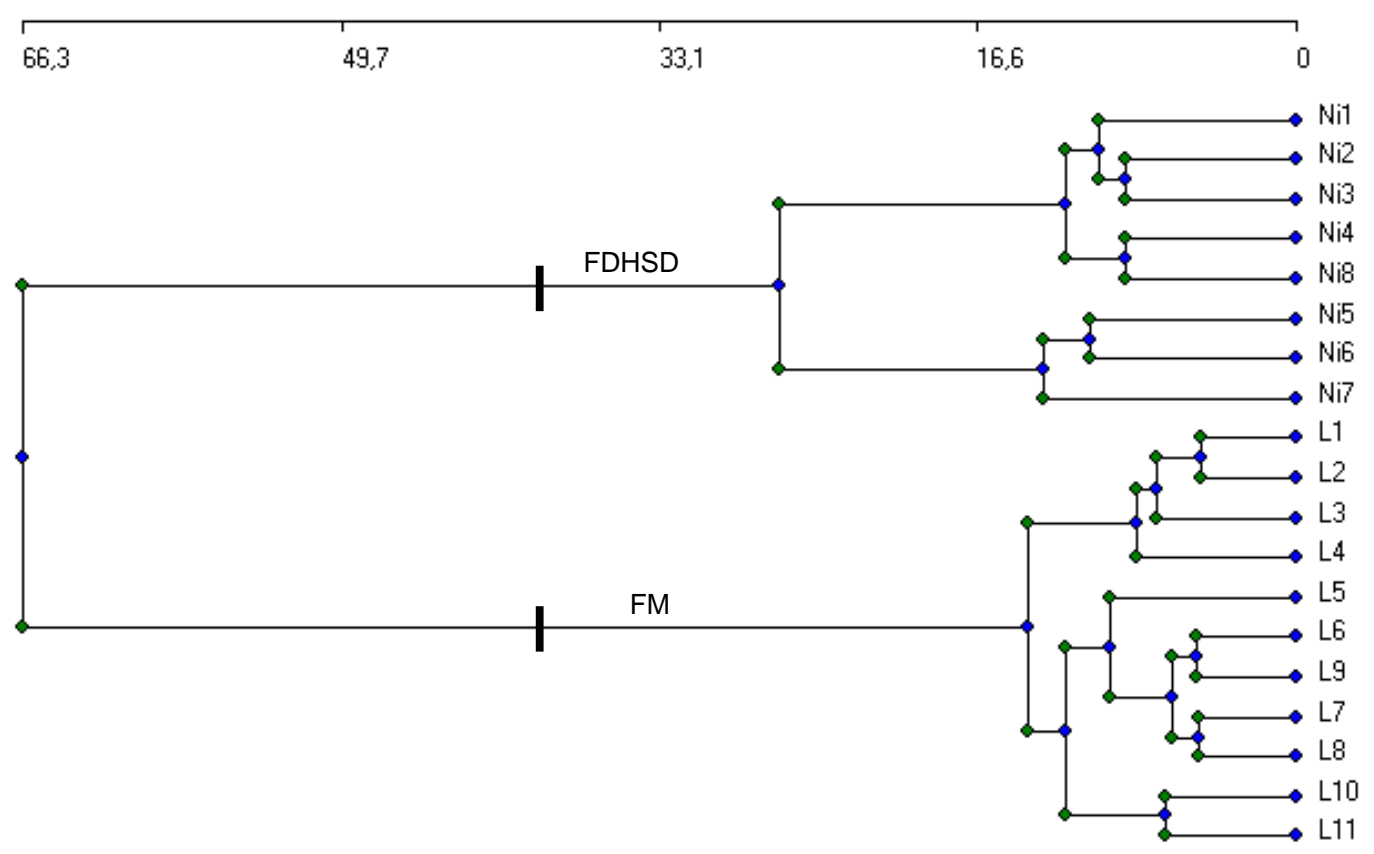

Figure 2 : Dendrogramme issu de la classification hiérarchique ("cluster analysis", méthode de Ward) d'une matrice de 19 relevés floristiques et 211 espèces végétales montrant les groupements végétaux. FDHSD = forêt dense humide semi-décidue de Niaouli, FM = forêt marécageuse de Lokoli.

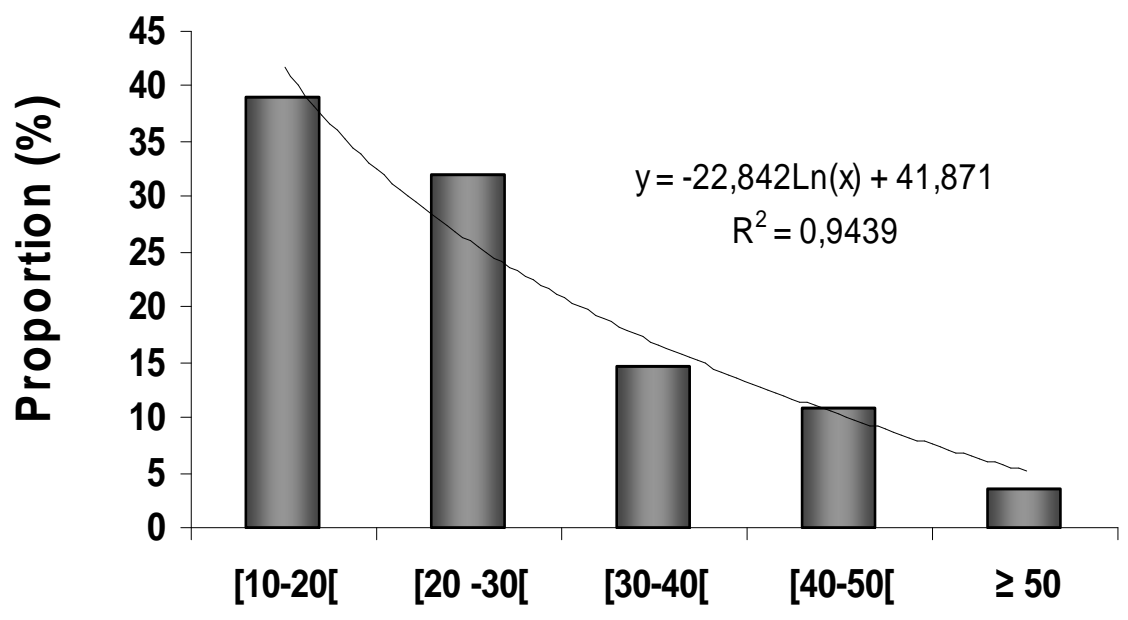

\section{Classes de diamètre $(\mathrm{cm})$}

Figure 3: Structure diamétrique des ligneux dans la forêt marécageuse de Lokoli. 


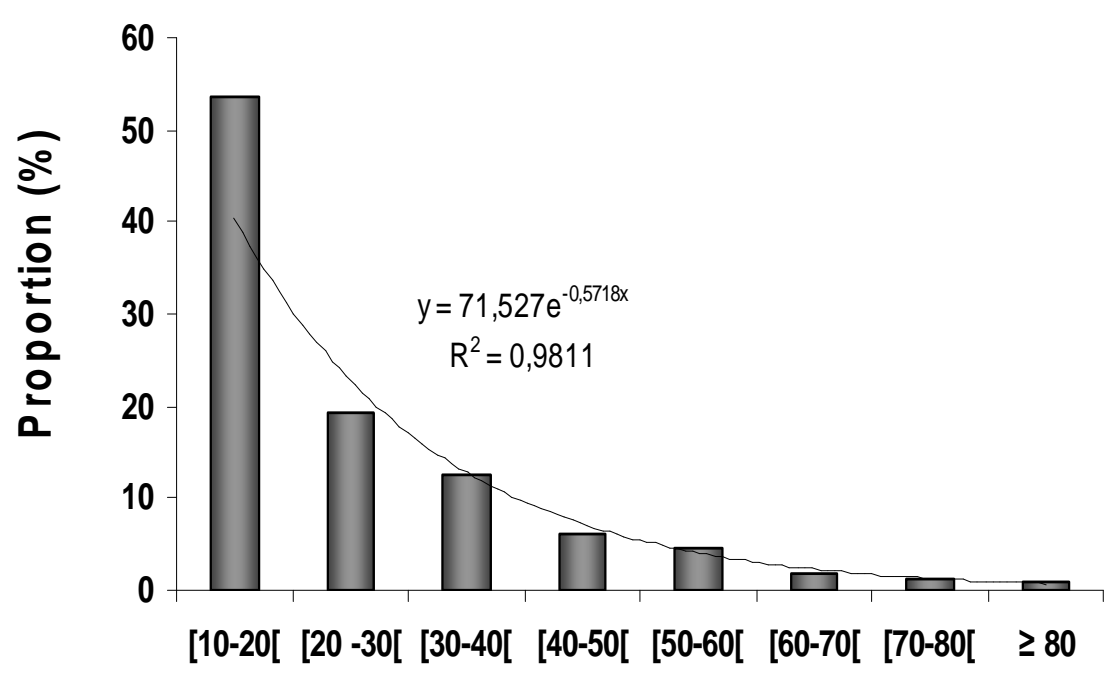

Classes de diamètre $(\mathrm{cm})$

Figure 4 : Structure diamétrique des ligneux dans la forêt de Niaouli.

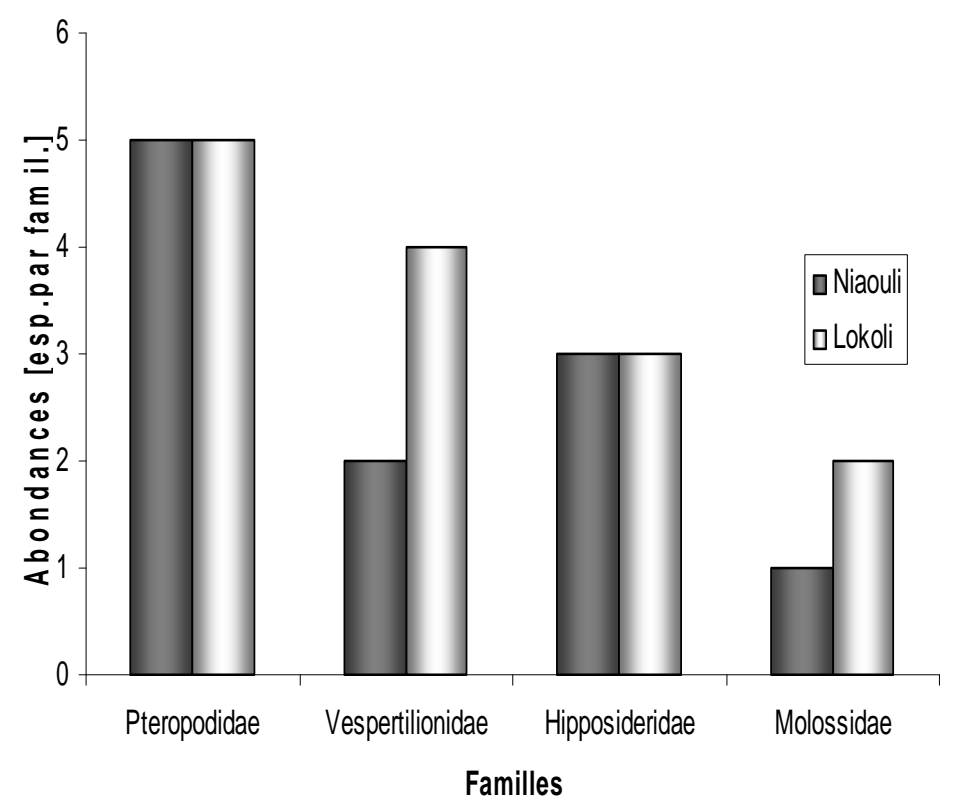

Figure 5 : Diversité des familles de chiroptères dans les forêts de Niaouli et de Lokoli. 


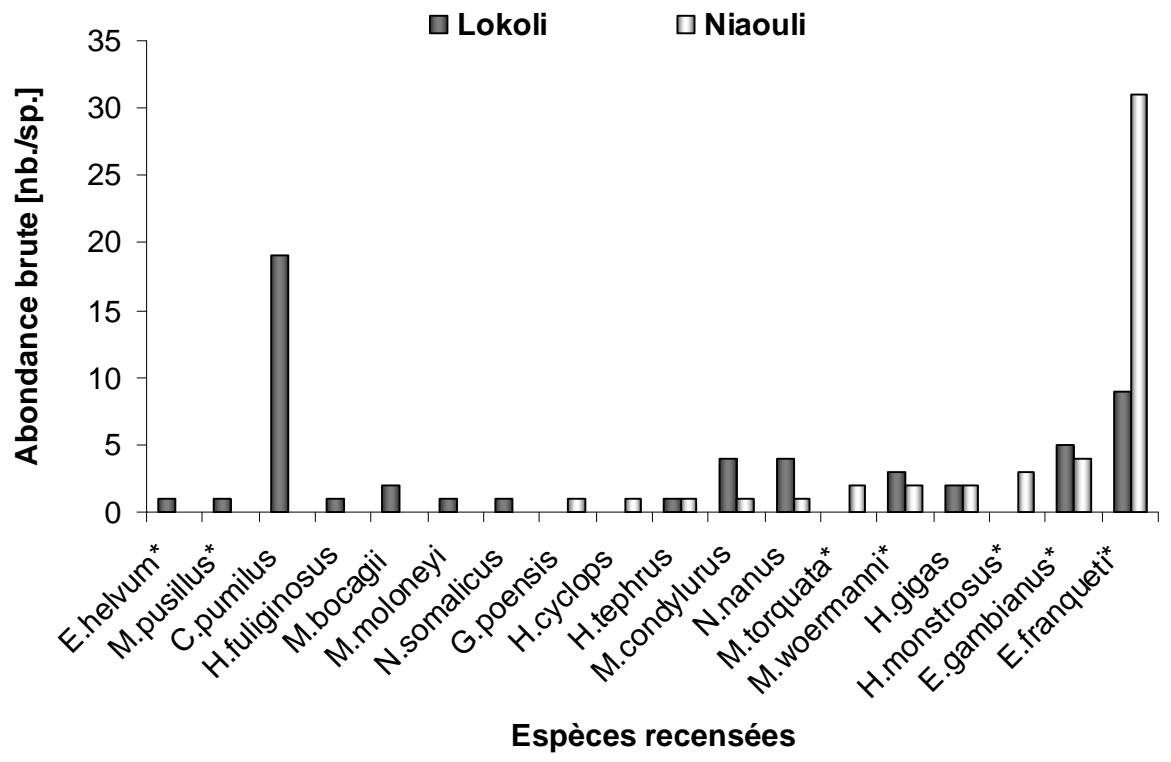

Figure 6 : Diversité comparée des chiroptères entre les forêts de Lokoli et de Niaouli. Frugivores marqués par une astérisque. Frugivores marqués avec astérisque $; \mathrm{Nb}=$ nombre, $\mathrm{sp}=$ espèce.

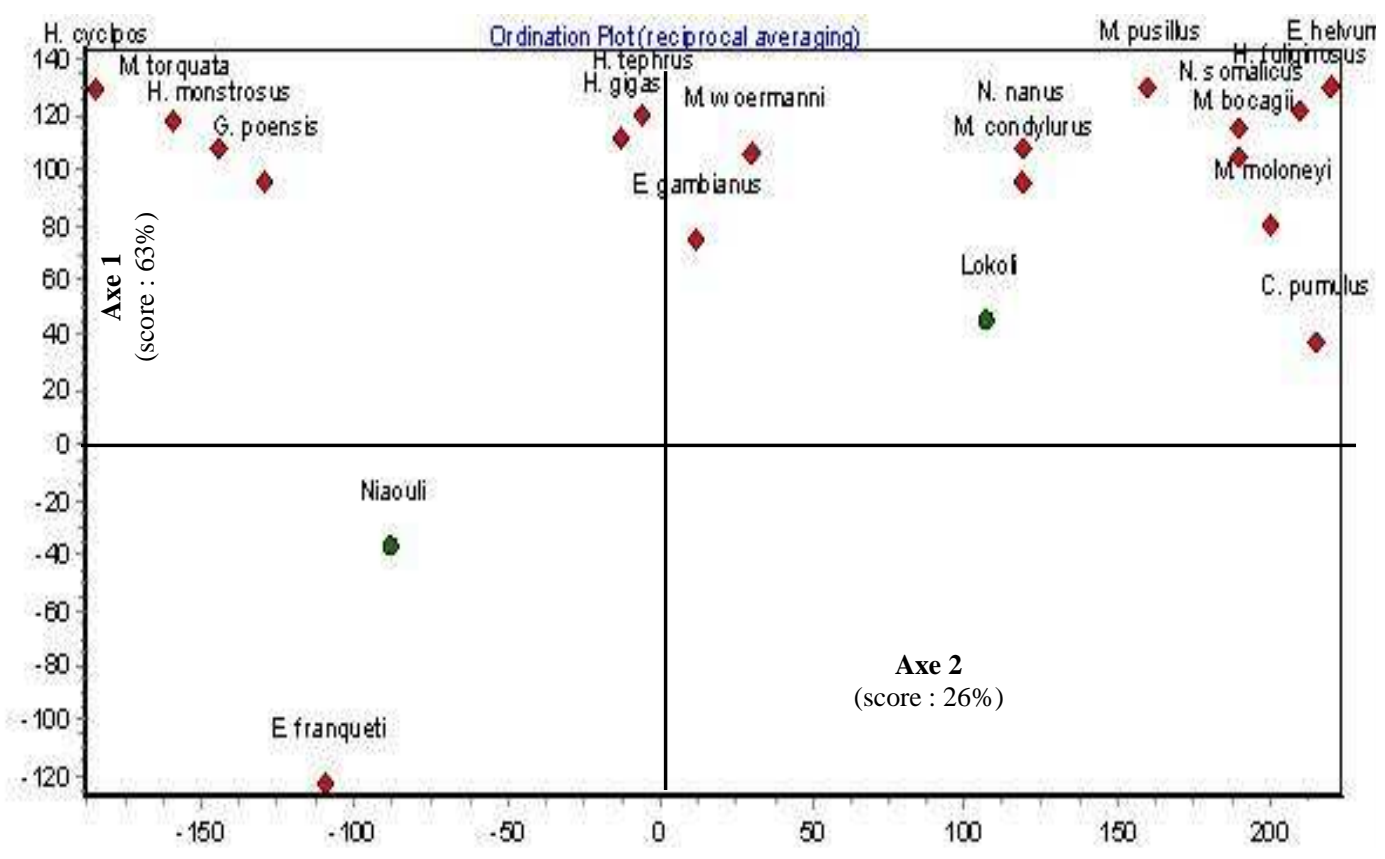

Figure 7 : Typologie des communautés de chiroptères entre les forêts de Niaouli et de Lokoli au moyen d'une ordination. 
Tableau 1: Diversité des chiroptères dans les forêts de Niaouli et de Lokoli.

\begin{tabular}{|c|c|c|c|c|}
\hline Familles & Species & Niaouli & Lokoli & $\begin{array}{l}\% \text { de la diversité nationale } \\
\text { connue }\end{array}$ \\
\hline \multirow[t]{7}{*}{ Pteropodidae } & Eidolon helvum (Kerr, 1792) & & $\mathrm{X}$ & \multirow{7}{*}{$\begin{array}{l}\text { Cette famille } 70 \% \text { des } \\
\text { espèces de cette famille } \\
\text { connues à ce jour. }\end{array}$} \\
\hline & Epomophorus gambianus (Ogilby, 1835) & $\mathrm{X}$ & $X$ & \\
\hline & Epomops franqueti (Tomes, 1860) & $\mathrm{X}$ & $\mathrm{X}$ & \\
\hline & Hypsignathus monstrosus (H. Allen, 1861) & $\mathrm{X}$ & & \\
\hline & Megaloglosus woermanni Pagenstecher, 1885 & $\mathrm{X}$ & $\mathrm{X}$ & \\
\hline & Micropteropus pusillus (Peters, 1867) & & $\mathrm{X}$ & \\
\hline & Myonycteris torquata (Matschie, 1899) & $\mathrm{X}$ & & \\
\hline \multirow[t]{5}{*}{ Vespertilionidae } & Glauconycteris poensis (GRAY, 1842) & $\mathrm{X}$ & & \multirow{5}{*}{$\begin{array}{l}27,8 \% \text { des espèces de cette } \\
\text { famille connues à ce jour. }\end{array}$} \\
\hline & Mimetellus moloneyi (Thomas, 1891) & & $X^{*}$ & \\
\hline & Myotis bocagii (Peters, 1870) & & $\mathrm{X}$ & \\
\hline & Neoromicia nanus (Thomas, 1901) & $\mathrm{X}$ & $\mathrm{X}$ & \\
\hline & Neoromicia somalicus (Thomas, 1901) & & $\mathrm{X}$ & \\
\hline \multirow[t]{4}{*}{ Hipposideridae } & Hipposideros gigas (Wagner, 1845) & $\mathrm{X}$ & $\mathrm{X}$ & \multirow{4}{*}{$\begin{array}{l}50 \% \text { des espèces de cette } \\
\text { famille connues à ce jour. }\end{array}$} \\
\hline & Hipposideros fuliginosus (Temminck, 1853) & & $X^{*}$ & \\
\hline & Hipposideros tephrus (Cabrera, 1906) & $X^{*}$ & $\mathrm{X}$ & \\
\hline & Hipposideros cyclpos (Temminck, 1853) & $\mathrm{X}$ & & \\
\hline \multirow[t]{2}{*}{ Molossidae } & Chearephon pumulus (Cretzschmar, 1830) & & $\mathrm{X}$ & \multirow{2}{*}{$\begin{array}{l}33,3 \% \text { des espèces de cette } \\
\text { famille connues à ce jour. }\end{array}$} \\
\hline & Mops condylurus (A. Smith, 1833) & $\mathrm{X}$ & $\mathrm{X}$ & \\
\hline Total & 18 espèces dont respectivement & 11 & 14 & Environ $34 \%$ des espèces. \\
\hline
\end{tabular}


La forêt de Niaouli qui abrite une station de recherche bénéficie d'un statut de conservation différente de la forêt marécageuse communautaire de Lokoli qui ne bénéficie d'aucun statut juridique de protection et qui est encore sous l'emprise des communautés riveraines qui prélèvent du bois énergie et bois d'œuvre, du matériel végétal à des fins de médecine traditionnelle et surtout exploitent le raphia (Raphia hookeri) pour l'extraction du vin, une filière qui occupe une bonne partie des actifs agricoles des villages voisins (Adomou et al., 2009). Cette situation a conduit à dégrader la forêt de Lokoli par endroit qui, avec les troués dans des portions continues de formations denses créent une certaine hétérogénéité d'habitats favorable à la diversité des chiroptères (Fahr et al., 2006). Le rôle et l'importance des forêts communautaires dans la conservation de la faune sauvage terrestre dans d'autres localités du Bénin sont aussi rapportés (Akouèhou et al., 2010). La différence entre les deux forêts étudiées comme le montre la typologie des communautés végétales est confirmée également par la structure des communautés des chiroptères. Toutefois, une certaine ressemblance existe du fait que le nombre d'espèces communes aux deux forêts atteignant $39 \%$ de la richesse spécifique totale. En effet, la forêt de Niaouli se subdivise en deux blocs dont un sur le plateau et l'autre dans le bas-fond. Megaloglosus woermanni, une espèce commune aux deux forêts a été capturée tant dans cette dernière zone de la forêt de Niaouli que dans la forêt marécageuse de Lokoli. Cette espèce qui se nourrit à dominance de nectar est connue comme une espèce de forêt (Bergmans, 1997; Fahr et Ebigbo, 2003) mais aussi des forêts dégradées et de leurs environs où sont installées des champs de plantation de bananerais (Mickleburgh et al., 1992). Epomops franqueti, capturée dans ces deux forêts était rencontrée à Niaouli tant dans le bas-fond que sur le plateau, une formation moins dense comme Bergmans (1989) l'avait aussi rapporté d'habitats semblables. Hipposideros gigas et Hipposideros tephrus connues comme des espèces inféodées aux grottes mais aussi aux forêts tropicales (Hutson et al., 2001) ont aussi été capturées dans les deux forêts au cours de la présente étude. La quasi-totalité des espèces capturées étaient connues comme dépendantes des forêts denses démontrant l'appartenance de ces deux habitats à la liste des reliques de forêts denses (Akoègninou, 1998; Adomou, 2005 ; Adomou et al., 2007) du Sud du Benin. Myotis bocagii, capturée seulement dans la forêt marécageuse de Lokoli est une espèce assez rare et attachée à la présence de l'eau (Hutson et al., 2001) si bien que les quelques mentions de cette espèce pour le Bénin ont été au niveau de la Rivière Pendjari dans l'extrême Nord-Ouest (Green, 1983; Haquart et Rombaut, 1995 ; Djossa, 2007).

Importance des îlots de forêts denses humides pour la conservation des chiroptères au Sud du Bénin

Le Sud du Bénin se situe dans le Dahomey-Gap (Salzmann and Hoelzmann, 2005), qui est une région connue pour sa pauvreté en formations forestières humides (Adomou et al., 2007 ). La présence dans cette région des forêts de Niaouli et de Lokoli, toutes deux reliques de forêts denses humides, servent de refuge aux espèces animales dépendantes des formations forestières denses. La majorité des espèces de chauves-souris capturées au cours de la présente étude sont des espèces de forêt dense humide (Hutson et al., 2001; Mickleburgh et al., 2004). Par ailleurs, E. franqueti et $H$. cyclops avaient été capturées en 1968 par Robbins (1980) de Kpodavé dans le Mono, une localité qui abritait une formation forestière dense. Kpodavé est situé à environ $40 \mathrm{~km}$ du côté Ouest de Niaouli. E. franqueti, M. woermanni et $H$. cyclops étaient aussi rapportées de la forêt de la Lama (Voglozin, 2005), une autre formation dense située à environ $25 \mathrm{~km}$ plus au Nord de Niaouli (Djossa et al., 2008). Ce qui montre bien que les reliques de forêts denses qu'on retrouve dans ce hiatus phytogéographique dit Dahomey-Gap que sont les forêts de Niaouli et de Lokoli, objet de la présente étude, permettent visiblement 
de conserver ces espèces dépendantes de forêts. Epomops, Hypsignathus et Megaloglossus avaient été mentionnées par Booth (1954) comme des genres se retrouvant seulement au niveau des blocs Ouest et Est de forêts denses humides qui délimitent le Dahomey-Gap et dans le même ordre d'idée, Bergmans (1997) rapportait que le DahomeyGap était une barrière pour Hypsignathus et Megaloglossus ce que les résultats actuels ne supportent pas. La présence de ces espèces dans le Sud du Bénin qui fait partie du Dahomey Gap est alors imputable à la présence des reliques de forêts denses humides.

Dans la liste des espèces capturées au cours de la présente étude, Chaerephon pumilus et Mops condylurus sont toutes deux espèces qu'on retrouve aussi dans des habitations humaines (Hutson et al., 2001), donc leur présence est un signe de dégradation ou de perturbations profondes par les activités anthropiques. Il se pose alors le problème de la conservation des ces habitats rares surtout dans cette région du Bénin, et un moyen efficace serait de les ériger en parcs nationaux (Akouèhou et al., 2010) afin que les moyens idoines soient mis en place pour leur conservation pour les générations actuelles et futures.

Nouvelles espèces de chiroptères pour le Bénin

Mimetillus moloneyi, Hipposideros fuliginosus et Hipposideros tephrus sont recensées pour la première fois pour le Bénin et cela enrichit la liste des chauves-souris du Bénin de $8 \%$ faisant passer le nombre d'espèces de 51 à 54 . En terme de distribution des nouvelles espèces, Hipposideros fuliginosus était connue dans la Sous-région ouest africaine de la Serra Léone, du Libéria, du Ghana et du Togo. Cette étude permet donc de l'étendre au Bénin. Mimetillus moloneyi était connue du Ghana, du Libéria, du Nigeria, de la Sierra Léone et du Togo. Le gap dans sa distribution vient donc d'être comblé avec sa présence rapportée au Bénin. Hipposideros tephrus dont la distribution couvre seulement l'Afrique de l'Est et de l'Ouest (Vallo et al.,
2008) avait été recensée dans la forêt de Nsukka, Etat de Anambra au Nigeria (Okafor, 1988). La présente étude intègre donc le Bénin dans son aire de distribution dans la sous région ouest africaine.

\section{Conclusion}

La présente étude montre l'importance et la place des forêts de Niaouli et de Lokoli dans la conservation de la faune en général et des chiroptères en particulier, surtout dans cette partie du Bénin où les aires protégées légalement conservées sont rares. Les pressions anthropiques dues à la densité élevée de la population humaine dans cette région justifient plus que jamais la nécessité de repenser la conservation de ces habitats particuliers pour augmenter les chances de ne pas perdre la diversité biologique qui y est attachée avant même de la connaître.

\section{REMERCIEMENTS}

Nous exprimons tous nos remerciements à tous ceux qui, de près ou de loin, ont facilité la conduite des travaux de terrain, aux habitants du village de Lokoli pour nous avoir accueilli et hébergé tout au long des collectes de données, Vigan Jean-Luc et Guèdègbé Mathurin pour nous avoir assisté lors des travaux de terrain.

\section{BIOBLIOGRAPHIE}

Adomou AC, Mama A, Missikpode R, Sinsin B. 2009. Cartographie et caractérisation floristique de la forêt marécageuse de Lokoli (Bénin). Int. J. Biol. Chem. Sci., 3(3): 492-503.

Adomou AC. 2005. Vegetation patterns and environmental gradient in Benin: Implications for biogeography and conservation. $\mathrm{PhD}$ thesis, Wageningen University, the Netherlands, p 136.

Adomou CA., Yédomonhan H, Sinsin B, Van der Maesen LJ. 2007. Distribution des aires protégées et conservation de la flore en république du Bénin. In Quelles aires protégées pour l'Afrique de l'Ouest? Fournier A, Sinsin B, Mensah AG (eds). 
Séminaire de Parakou (Bénin), 14-19 avril 2003. 342-352.

Agosta SJ. 2002. Habitat use, diet and roost selection by the Big Brown Bat (Eptesicus fuscus) in North America: a case for conserving an abundant species. Mammal Rev., 32(2): 179-198.

Akoègninou $\mathrm{A}$, van der Burg $\mathrm{WJ}$, van der Maesen LJG. 2006. Flore Analytique du Bénin. Backhuys Publishers: Wageningen.

Akoègninou A, 1998. Les forets denses humides semi-décidues du Sud-Bénin. Journal de la Recherche Scientifique de l'Université du Bénin, 2(1): 125-131.

Akouèhou GS, Djossa BA, Ahononga FC, Awessou BK, Sinsin BA. 2010. Role of community forest reserves in wildlife conservation in Benin, West Africa. Int. J. Biol. Chem. Sci., 4(4): 1318-1327.

Bergmans W. 1989. Taxonomy and biogeography of African fruit bats (Mammalia, Megachiroptera) 2. The genera Micropteropus Matschie, 1899, Epomops Gray, 1870, Hypsignathus H.Allen, 1861, Nanonycteris Matschie, 1899, and Plerotes Andersen, 1910. Beaufortia, 39(4): 89-153.

Bergmans W. 1997. Taxonomy and biogeography of African fruit bats (Mammalia, Megachiroptera). 5. The genera Lissonycteris Andersen, 1912, Myonycteris Matschie, 1899 and Megaloglossus Pagenstecher, 1885; general remarks and conclusions; annex: Key to all species. Beaufortia, 47(2): 1190.

Bergmans W. 2002. Les chauves-souris (Mammalia, Chiroptera) de Bénin. Compte rendu préliminaire. IUCN. Amsterdam. p 41.

Booth AH. 1954. The Dahomey Gap and the mammalian fauna of the West African Forest. Rev. Zool. Bot. Afr., 50(3-4): 305314.

Braun-Blanquet J. 1932. Plant Sociology: The Study of Plant Communities. McGray Hill: London, United Kingdom.
Djossa AB. 2007. Gestion des essences agroforestières spontanées et rôles des roussettes dans la dispersion de leurs semences dans la Réserve de Biosphère de la Pendjari (Bénin). Thèse de doctorat. FLASH, Université d'Abomey-Calavi, p 178.

Djossa BA, Sinsin BA, Kalko EKV, Jakob F. 2008. Inventory of bat species of Niaouli Forest, Benin, and its bearing on the significance of the Dahomey Gap as a zoogeographic barrier. African Bat Conservation News, 15: 4-6.

Fahr J, Djossa BA, Vierhaus H. 2006. Rapid assessment of bats (Chiroptera) in Déré, Diécké and Mt. Béro classified forests, Southeastern Guinea; including a review of the distribution of bats in Guinée Forestière. In Rapid Biological Assessment of Three Classified Forests in Southeastern Guinea, Wright HE, McCullough J, Alonso LE, Diallo MS (eds). A. RAP Bulletin of Biological Assessment $40 . \quad$ Conservation International: Washington, DC; 69-81.

Fahr J, Ebigbo NM. 2003. A conservation assessment of the bats of the Simandou Range, Guinea, with the first record of Myotis welwitschii (Gray, 1866) from West Africa. Acta Chiropterologica, 5(1): 125-141.

FAO. 2005. Evaluation des Ressources Forestières Mondiales 2005. FAO : Rome, Italie; 320.

Fenton MB. 1997. Science and the conservation of bats. Journal of Mammalogy, 78: 1-14.

Frontier S, Pichod-Viale D. 1991. Ecosystème: Structure, Fonctionnement, Evolution. Collection d'Ecologie. Masson: Paris.

Frontier S. 1983. Choix et contraintes de l'échantillonnage écologique. In Stratégie d'Echantillonnage en Ecologie, Frontier $\mathrm{S}$ (éd). Les Presses de l'Univ. de Laval: Laval; 3-62.

Green A. 1983. Rodents and bats of Arli and Pendjari National Parks, Upper Volta and Benin. Nigerian Field, 47(4): 167-184. 
Haquart A, Rombaut D. 1995. Contribution à l'inventaire des Chiroptères du Parc National et de la zone cynégétique de la Pendjari. Bénin. $52 \mathrm{p}+$ annexes Projet Pendjari. Cotonou. Bénin.

Hawthorne WD, Jongkind C. 2006. Woody plants of Western African Forests: A Guide to the Forest Trees, Shrubs and Lianes from Senegal to Ghana. Kew Publishing: Royal Botanic Gardens: Kew; 1023.

Hayman RW, Hill JE. 1971. Order Chiroptera, in: The Mammals of Africa, an Identification Manual, Meester J, Setzer HW (eds). Smithsonian Institution: Washington, D.C. ; 1-73.

Henle K, Davies KF, Kleyer M, Margules C, Settele J. 2004. Predictors of species sensitivity to fragmentation. Biodiversity and Conservation, 13: 207-251.

Henle K, Streit B. 1990. Kritische Beobachtungen zum Artenrückgang bei Amphibien und Reptilien und zu dessen Ursachen. Natur und Landschaft, 65 : 347-361.

Hodgkison R, Balding ST, Kunz TH. 2004. Temporal Variation in the Relative Abundance of Fruit Bats (Megachiroptera: Pteropodidae) in Relation to the Availability of Food in a Lowland Malaysian Rain Forest. BIOTROPICA, 36(4): 522-533.

Houinato M, Sinsin B. 2000. La pression Agro-pastorale sur la zone riveraine de la Réserve de Biosphère de la Pendjari. TROPICULTURA, 18(3): 112-117.

Hutson AM, Mickleburgh SP, Racey PA. 2001. Microchiropteran bats: global status survey and conservation action plan, IUCN/SSC Chiroptera Specialist Group, IUCN, Gland, Switzerland and Cambridge, UK, $\mathrm{x}+\mathrm{p} 258$.

INSAE 2002. Troisième recensement général de la population et de l'habitat. Résultats définitifs: Tableaux statistiques Cotonou, Bénin, p 4.
Mickleburgh SP, Hutson AM, Racey AP. 1992. Old World Fruit Bats. An Action Plan for their Conservation. IUCN/SSC Chiroptera Specialist Group. Gland, Switzerland. p 261.

Okafor FC. 1988. Oochoristica agamae Baylis, 1919 (Eucestoda, Linstowiidae) in one reptile and two bat species from Nsukka (Anambra State, Nigeria). Misc. Zool., 12.

Pierson ED. 1998. Tall trees, deep holes, and scarred landscapes: conservation biology of North American bats. In Bat Biology and Conservation, Kunz TH, Racey PA (ed). Smithsonian Institution Press: Washington, DC; 309-325.

Robbins CB. 1978. The Dahomey gap - a reevaluation of its significance as a faunal barrier to West African forest mammals. Bull. Carnegie Mus. nat. Hist., 6: 168174.

Salzmann U, Hoelzmann P. 2005. The Dahomey Gap: An abrupt climatically induced rain forest fragmentation in West Africa during the late Holocene. The Holocene, 15(2): 190-199.

Shannon CE. 1948. A mathematical theory of communications. Bell Syst. Techn. J., 27: 623-656.

Vallo P, Guillen-Servent A, Benda P, Pires DB, Koubek P. 2008. Variation of mitochondrial DNA in the Hipposideros caffer complex (Chiroptera: Hipposideridae) and its taxonomic implications. Acta Chiropterologica, 10(2): 193-206.

Voglozin NCA. 2005. Influences des systèmes agro-forestiers sur la diversité des communautés de chauves-souris dans la forêt classée de la Lama. DEA-Thesis, Faculté des Sciences Agronomiques, Université d'Abomey-Calavi, Bénin, p 65. 